\title{
A cucumber NAM domain transcription factor promotes pistil development in Arabidopsis
}

\author{
Jian Pan ${ }^{1}$, Hai-Fan Wen ${ }^{1}$, Wen-Hui Lin ${ }^{2^{*}}$ and Jun-Song Pan ${ }^{1 *}$
}

\section{Introduction}

Sexual reproduction is a fundamentally important biological process in nature. Unisexual flowers provide a widely used system for studying plant sex organ differentiation. Cucumber (C. sativus L.) generates both unisexual and bisexual flowers, and the sex type is mainly controlled by several ethylene synthases, i.e., CsACS2, CsACS1G, CsACS11, and CsACO2 (Boualem et al., 2008; Martin et al., 2009; Boualem et al., 2015; Chen et al., 2016). The unisexual flowers typically result from the selective suppression of one of the two genders in flower development stages 6-7 (Bai et al., 2004). Meanwhile, if the suppression is deactivated, caused by loss-offunction CsACS2 (CmACS7 in melon), functional stamens develop to make the flower bisexual (Li et al., 2009). The prior studies have demonstrated that an ethylene positive feedback regulation mediates by CsACS2 and CsERF31 is pivotal for cucumber female flower differentiation ( $\mathrm{Li}$ et al., 2012; Pan et al., 2018). However, the molecular mechanism of how the ethylene signal promotes female organ development remains elusive. Here, we identified a cucumber putative NAM domain transcription factor, CsNAC2, that showed specific female organ expression. Ectopic expression of $C s N A C 2$ in Arabidopsis led to carpelloid stamens and decreased the ethylene accumulation, implying fine-tuned ethylene regulation post-sexual differentiation in cucumber. Our finding provides new clues to understand the regulatory mechanism of female organ differentiation.

\footnotetext{
* Correspondence: whlin@sjtu.edu.cn; jspan71@sjtu.edu.cn

${ }^{2}$ School of Life Sciences and Biotechnology, The Joint International Research Laboratory of Metabolic \& Developmental Sciences, Joint Center for Single

Cell Biology, Shanghai Jiao Tong University, Shanghai, China

'School of Agriculture and Biology, Shanghai Jiao Tong University, Shanghai, China
}

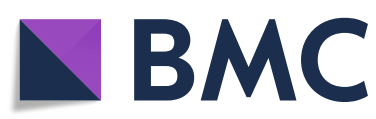

(0 The Author(s). 2021 Open Access This article is licensed under a Creative Commons Attribution 4.0 International License, which permits use, sharing, adaptation, distribution and reproduction in any medium or format, as long as you give appropriate credit to the original author(s) and the source, provide a link to the Creative Commons licence, and indicate if changes were made. The images or other third party material in this article are included in the article's Creative Commons licence, unless indicated otherwise in a credit line to the material. If material is not included in the article's Creative Commons licence and your intended use is not permitted by statutory regulation or exceeds the permitted use, you will need to obtain permission directly from the copyright holder. To view a copy of this licence, visit http://creativecommons.org/licenses/by/4.0/. The Creative Commons Public Domain Dedication waiver (http://creativecommons.org/publicdomain/zero/1.0/) applies to the data made available in this article, unless otherwise stated in a credit line to the data.

\section{Results}

Having identified the ethylene positive feedback regulation in the cucumber female flower determination (Pan et al., 2018; Pan et al., 2021), we next studied how highly accumulated ethylene promoted female organ differentiation. Based on our previous transcriptomic analysis in the shoot apexes of different genotypes in cucumber (Pan et al., 2018), we found a NAM domain transcription factor, CsNAC2, which had a specific high expression in gynoecious line. In this study, we tested the mRNA expression level of $C s N A C 2$ in the flower buds (before stage 7, according to (Bai et al., 2004)) of different genotypes, as gynoecious line, G12 (CsACS1G/ CsACS2); monoecy line, M12 (CsACS1/CsACS2); and hemaphrodite line, H34 (CsACS1G/csacs2). CsNAC2 showed a more significant high expression in the female bud in G12 (Fig. 1A), suggesting its function might relate to female flower development. Although $\operatorname{CsNAC2}$ displayed a relatively higher expression in $1 \mathrm{~mm}$ (before stage 7) predetermined female bud, the highest expression is in the $2 \mathrm{~mm}(\sim$ stage $8-2)$ female bud, which differs from most sex-determination genes in cucumber, like CsACS2, CsACS11 and CsACO2 (Fig. 1B) (Zhang et al., 2020). The higher expression in $2 \mathrm{~mm}$ female bud suggested that CsNAC2 might promote female organ primordium differentiation after the sex determination. In situ hybridization further showed that the transcripts of $C s N A C 2$ were specifically accumulated in both stamen and pistil primordia in the predetermined $1 \mathrm{~mm}$ bud; then only in the pistil primordium of the $2 \mathrm{~mm}$ female flower bud (Fig. 1C).

Given that ethylene has been widely accepted as a critical regulator of cucumber female flower development, we further tested the effect of ethylene-related treatments on the expression of $C s N A C 2$ on the gynoecious 


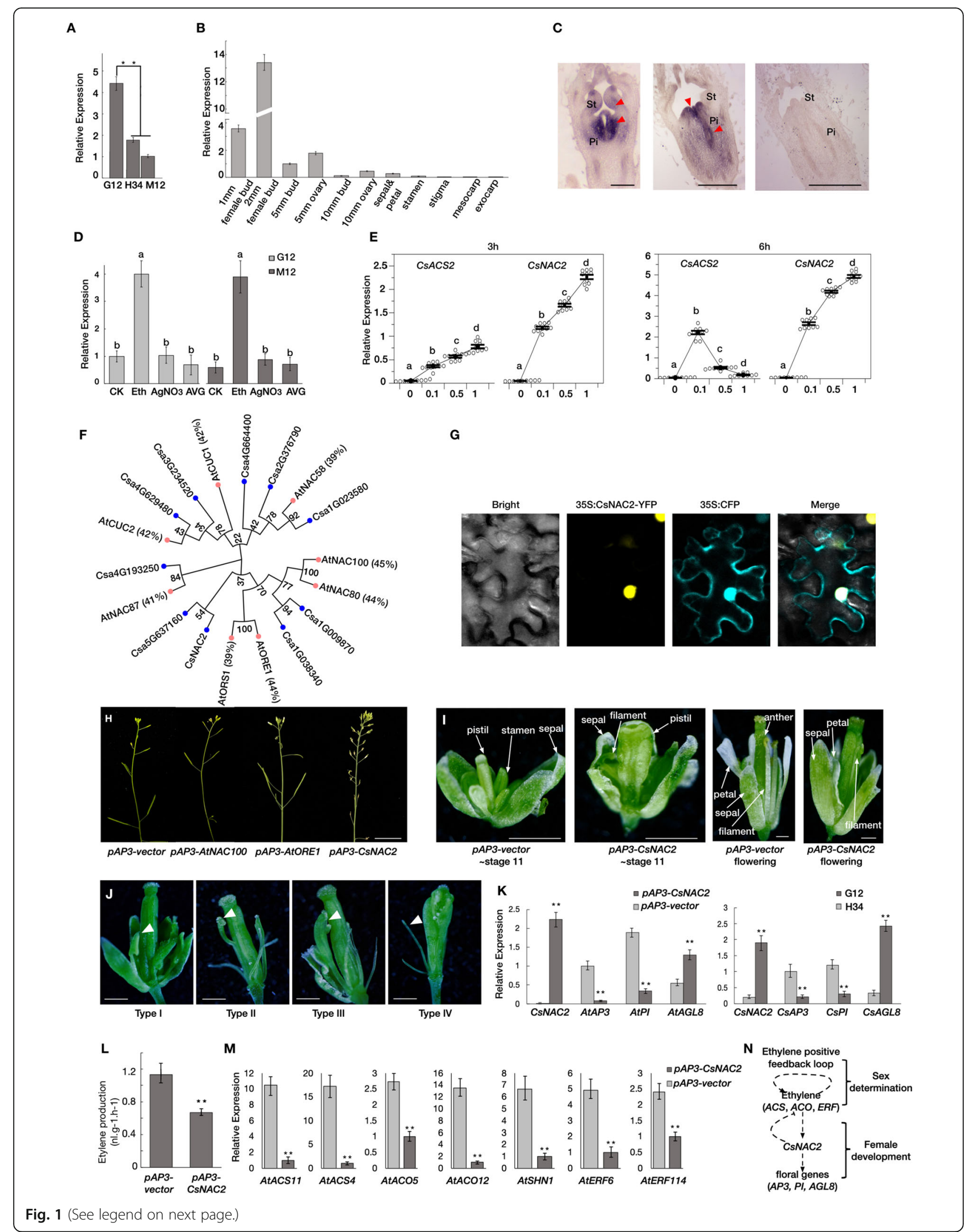


(See figure on previous page.)

Fig. 1 A Expression analysis of CSNAC2 in $1 \mathrm{~mm}$ buds of different genotype cucumber lines. Gynoecious line, G12 (CSACS1G/CSACS2); monoecy line, M12 (CSACS1/CSACS2); and hemaphrodite line, H34 (CSACS1G/CsaCs2). Error bars represent the SD from three biological replicates, and asterisks indicate significant differences in different treatments ( $t$-test, ${ }^{* *} p$ value $<0.01$ ). B Spatial-temporal expression patterns of $C s N A C 2$ in female development. Temporal expression patterns at different stages of female bud $(1,2,5$, and $10 \mathrm{~mm}$ buds were divided into $5 \mathrm{~mm}$ bud, 5 $\mathrm{mm}$ ovary, $10 \mathrm{~mm}$ bud, and 10-mm ovary, respectively) and spatial expression patterns of petal, stamen, stigma, mesocarp, and exocarp. Error bars represent the SD from three biological replicates. C In situ hybridization of CSNAC2 in gynoecy cucumber buds. Hybridization with antisenseprobe in stage 7 (left), stage 8-2 (middle). Red arrows indicate hybridization signal. Hybridization with sense-probe presents in right. St: stamen, Pi: pistil. Bar $=200 \mu \mathrm{m}$. D Expression patterns of CsNAC2 in shoot apices of the chemical treatments and control cucumber lines. Eth: ethephon, AgNO3: silver nitrate, AVG: aminoethoxyvinyl-glycine. Error bars represent the SD from three biological replicates. Different letters $(a, b)$ indicate significant differences (one-way ANOVA, $p$ value $<0.01$ ). E Expression level of CSNAC2 and CSACS2 in cucumber shoot apices of different concentrations treatment. Apical shoots of four-leaf stage seedlings were soaked in water containing 0, 0.1, 0.5, and $1.0 \mathrm{mM}$ ethephon for 3 and $6 \mathrm{~h}$, respectively. The circle indicates the expression of 10 biological replicates. The letters indicate the difference between each other $p$ value $<0.01$ in $(a, b),(b, c)$ and $(c, d)$ from $t$-test. F An unrooted phylogenetic tree of CsNAC2 from Arabidopsis and cucumber. The phylogenetic tree was constructed by the Neighbor-Joining method with 1000 bootstrap sampling. Bar represents patristic distances. G Subcellular localization of CSNAC2. Subcellular localization of CSNAC2-YFP fusion proteins in tobacco leaves. 35S:CFP indicates a marker for nuclear localization signal. H Phenotype result of Arabidopsis transgenic individual. Phenotypes of transgenic plants are T2 generation. All transgenic plants in different individual lines share the same phenotype, and only one line shows in result. I Floral organs phenotype of Arabidopsis transgenic individual. Flower bud phenotype of $P A P 3$-vector and pAP3-CSNAC2 in stage 11 (left) and flowering time (right). Bar $=0.2 \mathrm{~cm}$. $\mathbf{J}$ The four types phenotypes of pAP3-CSNAC2 flower buds. Type I, normal looking but non-functional stamen, with papillar tissue on the tips. Type II, carpelloid stamen fuse with carpel. Type III, a carpelloid stamen not fuse with carpel. Type IV, no stamens and abnormal carpel. The white arrows indicate the abnormal floral organs. Bars $=0.2 \mathrm{~cm}$. K Expression level of floral genes in transgenic Arabidopsis (left) and cucumber (right) plants. Error bars represent the SD from three biological replicates, and asterisks indicate significant differences in different treatments ( $t$-test, ${ }^{* *} p$ value $\left.<0.01\right)$. L Ethylene production in PAP3-CSNAC2 the transgenic Arabidopsis plants. Error bars represent the SD from four biological replicates, and asterisks indicate significant differences in different treatments (Student's $t$-test, ${ }^{* *} p$ value $<0.01$ ). $\mathbf{M}$ Expression level of ethylene related genes in the transgenic Arabidopsis. Error bars represent the SD from three biological replicates, and asterisks indicate significant differences in different transgenic plants ( $t$-test, ${ }^{* *} p$ value $<0.01$ ). N Model of CsNAC2 functions during the development of female flowers in Arabidopsis and cucumber. The ethylene signal activated by the positive feedback loop induces the expression of CSNAC2. CSNAC2 then promotes female organ development via activates floral genes in an unknown way, and represses the ethylene related genes to avoid an excessive ethylene effect in the flower bud

and monoecious cucumber lines. $\mathrm{AgNO}_{3}$ and AVG are known to interfere with ethylene action, and their application promoted the conversion of female flowers into male flowers. The results showed that the expression of CsNAC2 was not significantly changed in $\mathrm{AgNO} 3$ and AVG treatments. However, ethephon could induce the CsNAC2 expression in both gynoecious and monoecious lines (Fig. 1D). Therefore, we further tested the expression level of CsACS2, as a marker of female flower initiation, in different concentration ethephon treatments. We found CsACS2 was promoted under low level $(0.1$ $\mathrm{mM})$ and was inhibited under high level $(0.5$ and $1 \mathrm{mM})$ (Fig. 1E), which was consistent with the prior study (Li et al., 2012). However, the expression pattern of CsNAC2 was not followed that of CsACS2, and instead displayed a positive correlation with the ethylene concentration (Fig. 1E).

The result of phylogenetic analysis suggested that CsNAC2 has a relatively closer evolutionary relationship with AtNAC100 (45\%), AtORE1 (44\%), and AtNAC80 (44\%) in amino acid sequence (Fig. 1F). Subcellular localization analysis indicated that CsNAC2 was localized in the nucleus, which suggested a putative transcription factor (Fig. 1G). To determine if $C s N A C 2$ has a role in regulation of female organ differentiation, we constructed transgenic Arabidopsis plants with the organ-specific expression of $C s N A C 2$ under the control of an AtAP3 promoter, which specifically expressed in stamens. To verify the potentially distinguish function of CsNAC2 in promoting female development, we used the AtAP3 promoter drove AtNAC100, AtORE1 (ORESARA1) and the empty vector as controls (Fig. $1 \mathrm{H}$ ). The phenotype of the pAP3-CsNAC2 transgenic Arabidopsis plants showed that stamen and petal development were significantly altered, resulting in male sterility (23 out of 24 individuals) (Fig. 1I). For every individual among the 23 transgenic plants, four types of stamens could be classified according to their morphology. Type I ( 38\%): normal-looking but non-functional stamen, with papillar tissue on the tips. Type II $(\sim 21 \%)$ : carpelloid stamen partially fuse with carpel. Type III ( 29\%): a carpelloid stamen did not fuse with the carpel. Type IV $(\sim 12 \%)$ : no stamens and abnormal carpel (Fig. 1J). We performed pollination tests to confirm whether CsNAC2 expression affected the pistil development and found that the transgenic Arabidopsis plants could generate fertile offspring. The above results indicated that the ectopic expression of $C s N A C 2$ with $A P 3$ promoter promotes female organ differentiation, which is conserved with the condition in cucumber. The B gene, i.e., AP3 and PI, are known as the ruler of stamen and petal development (Bowman et al., 1991). Thus, we tested the expression level of AtAP3 and AtPI in pAP3-CsNAC2 Arabidopsis plants and found that both of them were significantly downregulated. This is consistent with reduced expression of CsAP3 and CsPI in gynoecious (G12) vs. hermaphrodite 
(H34) cucumber lines (Fig. 1K). Notably, AtAGL8, which controls the carpel development and fruit cell differentiation in Arabidopsis (Gu et al., 1998), showed two-folds upregulated in $p A P 3-C s N A C 2$ plants, and its ortholog CsAGL8 showed a similar upregulated expression in gynoecious cucumber line (G12 vs. H34 in Fig. 1K). The above results suggested that the regulatory mechanism of female organ promotion might be conserved in Arabidopsis and cucumber. Furthermore, we found CsNAC2 showed a DNA binding activation but cannot directly interact with the promoter of $A P 3, P I$, or $A G L 8$, via yeast one-hybrid and dual-luciferase assay (Supplemental Information Fig. S1).

Given the important role that ethylene plays in cucumber female flower development, we tested the ethylene productivity in the transgenic Arabidopsis flower buds. The results indicated ethylene production was significantly decreased in $p A P 3-C s N A C 2$ (Fig. 1L), suggesting that the expression of CsNAC2 suppressed the ethylene accumulation. We next investigated the expression level of several ethylene synthetases (ACSs and ACOs) and ethylene response factors (ERFs), and found that AtACS11, AtACS4, AtACO5, AtACO12, AtSHN1, AtERF6, and AtERF114 were significantly downregulated in $p A P 3-C s N A C 2$ plants (Fig. $1 \mathrm{M}$ ). Thus, our combined data suggest that $C s N A C 2$ is downstream of ethylene signal, and it may function in repressing male organ development and inducing female organ development, and regulating ethylene production (Fig. $1 \mathrm{~N}$ ).

\section{Discussion}

In the last decades, sufficient evidence has been reported to support ethylene promotes cucumber female flower development; however, we still gain little knowledge about the regulatory mechanism of how ethylene selectively promoting floral gene expression to produce the proper unisexual flower. In this study, we demonstrated the role of $C s N A C 2$ in responding to the upstream ethylene signal and promoting female organ development, meanwhile limiting the accumulation of ethylene produce (Fig. 1N). This result is consistent with the hypothesis that the amplified ethylene signal, which decides female initiation, should be limited to a certain level to avoid damaging organ primordium development ( $\mathrm{Li}$ et al., 2012; Pan et al., 2018). Besides, our results imply that CsNAC2 may act as a repressor to arrest the stamen development via indirectly down-regulating AP3 and PI in both cucumber and Arabidopsis (Fig. 1K), meanwhile promotes the pistil development. Our data provided evidence to link CsNAC2 expression pattern to female flower development in cucumber, and revealed a "carpelloid stamens" phenotype led by ectopic expressing CsNAC2 in Arabidopsis, which could help to understand the regulatory mechanism downstream of ethylene signaling in female floral organ development.

\section{Supplementary Information}

The online version contains supplementary material available at https://doi. org/10.1186/s43897-021-00013-w.

\section{Additional file 1.}

Acknowledgements

Not applicable.

Authors' contributions

J.P., and H.F.W. performed the research; J.S.P. and W.L. designed the project; J.P., and W.H.L. wrote the manuscript. All authors discussed the results and commented on the manuscript. The author(s) read and approved the final manuscript.

Funding

This work was supported by the Natural Science Foundation of China (grant nos. 31772308 and 31272185 ).

Availability of data and materials

All data generated or analyzed during this study are included in this published article.

\section{Declarations}

Ethics approval and consent to participate

Not applicable.

\section{Consent for publication}

Not applicable.

\section{Competing interests}

The authors declare that they have no competing interests.

Received: 10 February 2021 Accepted: 3 July 2021

Published online: 15 September 2021

\section{References}

Bai S-L, Peng Y-B, Cui J-X, Gu H-T, Xu L-Y, Li Y-Q, et al. Developmental analyses reveal early arrests of the spore-bearing parts of reproductive organs in unisexual flowers of cucumber (Cucumis sativus L.). Planta. 2004;220(2):230 40. https://doi.org/10.1007/s00425-004-1342-2.

Boualem A, Fergany M, Fernandez R, Troadec C, Martin A, Morin H, et al. A conserved mutation in an ethylene biosynthesis enzyme leads to Andromonoecy in melons. Science. 2008;321(5890):836-8. https://doi.org/1 $0.1126 /$ science. 1159023

Boualem A, Troadec C, Camps C, Lemhemdi A, Morin H, Sari M-A, et al. A cucurbit androecy gene reveals how unisexual flowers develop and dioecy emerges. Science. 2015;350(6261):688-91. https://doi.org/10.1126/science.aa c8370.

Bowman JL, Smyth DR, Meyerowitz EM. Genetic interactions among floral homeotic genes of Arabidopsis. Dev Camb Engl. 1991;112:1-20.

Chen H, Sun J, Li S, Cui Q, Zhang H, Xin F, et al. An ACC oxidase gene essential for cucumber carpel development. Mol Plant. 2016;9(9):1315-27. https://doi. org/10.1016/j.molp.2016.06.018.

Gu Q, Ferrándiz C, Yanofsky MF, Martienssen R. The FRUITFULL MADS-box gene mediates cell differentiation during Arabidopsis fruit development. Dev Camb Engl. 1998;125:1509-17.

Li Z, Huang S, Liu S, Pan J, Zhang Z, Tao Q, et al. Molecular isolation of the M gene suggests that a conserved-residue conversion induces the formation of bisexual Flowers in cucumber plants. Genetics. 2009;182(4):1381-5. https:// doi.org/10.1534/genetics.109.104737.

Li Z, Wang S, Tao Q, Pan J, Si L, Gong Z, et al. A putative positive feedback regulation mechanism in CSACS2 expression suggests a modified model for sex determination in cucumber (Cucumis sativus L.). J Exp Bot. 2012;63(12): 4475-84. https://doi.org/10.1093/jxb/ers123. 
Martin A, Troadec C, Boualem A, Rajab M, Fernandez R, Morin H, et al. A transposon-induced epigenetic change leads to sex determination in melon. Nature. 2009;461 (7267):1135-8. https://doi.org/10.1038/nature08498.

Pan J, Wang G, Wen H, Du H, Lian H, He H, et al. Differential gene expression caused by the $\mathrm{F}$ and $\mathrm{M}$ loci provides insight into ethylene-mediated female flower differentiation in cucumber. Front Plant Sci. 2018;9:1091. https://doi. org/10.3389/fpls.2018.01091.

Pan J, Wen H, Chen G, Lin W, Du H, Chen Y, et al. A positive feedback loop mediated by CsERF31 initiates female cucumber flower development: ETHYLENE RESPONSE FACTOR31 mediates a positive feedback loop that initiates female cucumber flower development. Plant Physiol. 2021;186(2): 1088-1100

Zhang H, Li S, Yang L, Cai G, Chen H, Gao D, et al. Gain-of-function of the 1-aminocyclopropane-1-carboxylate synthase gene ACS1G induces female flower development in cucumber gynoecy. Plant Cell. 2020;33(2):306-21. https://doi.org/10.1093/plcell/koaa018.

\section{Publisher's Note}

Springer Nature remains neutral with regard to jurisdictional claims in published maps and institutional affiliations.

Ready to submit your research? Choose BMC and benefit from:

- fast, convenient online submission

- thorough peer review by experienced researchers in your field

- rapid publication on acceptance

- support for research data, including large and complex data types

- gold Open Access which fosters wider collaboration and increased citations

- maximum visibility for your research: over $100 \mathrm{M}$ website views per year

At BMC, research is always in progress.

Learn more biomedcentral.com/submissions 